\title{
Kommentare
}

\section{Vom zeitgemäßen Sozialstaatsverständnis}

Vielen Jurastudenten - insbesondere der Reformstudiengänge - ist die "Sozialstaatsdiskussion « der bundesdeutschen Staatsrechtslehre als Arbeitsthema wohivertraut. Die Beschäftigung mit Ernst Forsthoffs Interpretationsansatz auf der Staatsrechtslehrertagung 1953, seiner strikten Entgegensetzung von Rechtsstaats- und Sozialstaatsprinzip ${ }^{\prime}$ mag manchen nachgerade als unverzichtbare Übung erscheinen. Wieweit man sich in diesem Zusammenhang auf die konträren Positionen Wolfgang Abendroths ${ }^{2}$ oder Hans Peter Ipsens von $1949^{\prime}$ und von $195 \mathrm{I}^{*}$ einläßt, hängt dann allerdings schon stark von der jeweiligen Bereitschaft $a b$, sich die Mitwirkung an der Verdrängungsarbeit des juristischen Meinungsbildungskartells zu versagen. Auch wenn man die Forsthoffsche Sozialstaatsinterpretation nicht als einen auf "alternative Sozialstaatsverständnisse zielenden restaurativen "Gegenstoß ${ }^{s}$ wertet, weiß man sich jedenfalls in der Kritik an Forsthoffs zentralen Thesen wie etwa der Ablehnung des Verfassungsrangs für das Sozialstaatsprinzip mit der »h. M. einig ${ }^{6}$. In einem wichtigen Punkt bestehen zwischen den Positionen Forsthoffs und der h. M. allerdings keine Differenzen: Eine Auslegung des Sozialstaatsprinzips entsprechend der "polemischen « Wortbedeutung von »sozial « im Sinne des Ziels einer Umverteilung der Güter ${ }^{7}$ bzw. der »Systemüberwindung “ ${ }^{8}$ lehnt man ab. Für die h. M. besteht kein Zusammenhang $z$ wischen dem Sozialstaatsgebot und der Sozialisierungsermächtigung des Art. Is GG; der vom Grundgesetz gemeinte »soziale Staat« sei keinesfalls ein sozialistischer, vielmehr ergäbe sich insbesondere aus den Grundrechten

1 E. Forsthoff, Begriff und Wesen des sozialen Rechtsstaates, VVDStRL 12 (1954), 8; auch abgedruckt in Ders. (Hrsg.), Rechtsstaatlichkeit und Sozialstaatlichkeit, Darmstadt $1968 \mathrm{~S}$. I65; aus der Fülle der Literatur, die sich mit dem Forsthoffschen Ansatz kritisch beschäftigt, vgl. nur: H.-H. Hartwich, Sozialstaatspostulat und gesellschaftlicher status quo, Köln u. a. 1970, S. 295 ff., 317 ff.; O. E. Kempen, Einleitung, in: Ders. (Hrsg.), Sozialstaatsprinzip und Wirtschaftsordnung, Frankfurt u. a. 1976, S. 7 (23 ff.); D. Suhr, Rechtsstaatlichkeit und Sozialstaatlichkeit, Der Staat 9 (1970),67, auch in: O. E. Kempen, a. a. O., S. 15 I (1 54 ff.); G. Stuby, Bürgerliche Demokratietheorien in der Bundesrepublik, in: R. Kühnl (Hrsg.), Der bürgerliche Staat der Gegenwart. Reinbek 1972, S. 87 (106 f.)

2 W. Abendroth, Zum Begriff des demokratischen und sozialen Rechtsstaates im Grundgesetz der Bundesrepublik Deurschland, in: Festschrift t. L. Bergsträßer, Düsseldorf 1965 , S. 279, sowie in W. Abend roth, Antagonistische Gesellschaft und politische Demokratie, Neuwied u. a. 1967, S. 109, O. E. Kempen a. a. O., S. 70 .

3 H. P. Ipsen, Über das Grundgeserz (Hamburger Universitätsrede 1949), Hamburg 1950, auch in: E. Forsthoff (Hrsg.), a. a. O., S. 16.

4 H. P. Ipsen, Enteignung und Sozialisierung, VVDStRL 10 (1952), 74.

s So H.-H. Hartwich, a. a. O., S. 295 .

6 Vgl. nur R. Herzog, in: Maunz-Dürig, Grundgesetz, Kommentar, Art. 20, Die Verfassungsentscheidung für die Sozialstaatlichkeit (Kommentierung von 1976/1980) Rdn. 2 u. 8 Fn. 2; K. Stern, Das Staatsrecht der Bundesrepublik Deutschland, Bd. I, München 1977, S. 688 f.

7 Vgl. E. Forsthoff, VVDStRL i 2,25 .

8 Y. Huh, Rechtsstaatliche Grenzen der Sozialstaatlichkeit? Der Staat 1979, 183 (192); Rupert Scholz,

Sozialstaat zwischen Wachstums- und Rezessionsgesellschaft, Karlsruhe 1981, S. 32. 
die verfassungsmäßige Garantie der marktwirtschaftlichen Ordnung in der Bundesrepublik 9 .

\section{Der Weg zur *h. M.*}

Die Herausbildung dieser in der Staatsrechtslehre weitverbreiteten Position läßt sich paradigmatisch an der Entwicklung der Auffassungen Hans Peter Ipsens zeigen. Auf der Staatsrechtslehrertagung I95 I sah Ipsen in der Verfassungsentscheidung für den Sozialstaat die Aufforderung zur Gestaltung der Sozialordnung, was in bezug auf die Eigentumsverfassung »die Neu- und Anders-Gestaltung der Eigentumsherrschaft bis hin zu seiner Neu-Verteilung "bedeute ${ }^{\text {to }}$. Mit Art. Is habe das Grundgesetz die Sozialisierung zum Inhalt seiner wirtschaftspolitischen Entscheidung gemacht und legalisiert". In seinem 1979 verfaßten Beitrag "Gebundene Sozialstaatlichkeit « wendet Ipsen sich gegen einen »ideologisch bestimmten Zugriff auf die Sozialstaatlichkeit ${ }^{12}$; und es erscheint ihm "mehr als fraglich, ob das Sozialstaatsprinzip eine staatliche Zuständigkeit und Ermächtigung hergibt, die Lage der Gesellschaft umverteilend und egalisierend gegenüber dem sozialen status quo grundlegend zu verwandeln $\aleph^{13}$. Ipsen zieht aus seinem »zeitgemäß ${ }^{2}$ veränderten Sozialstaatsverständnis auch Folgerungen hinsichtlich der Zulässigkeit politischer Parteiprogramme: "Ihre geforderte Ernsthaftigkeit verlangt mindestens ihre verfassungsrechtliche Zulässigkeit, weshalb sozialrevolutionäre Anforderungen an den Sozialstaat in den Parteiprogrammen keinen legitimen Standort haben ${ }^{24}$. Dies zielt weit über das herkömmliche Spektrum der vom »Verfassungsfeindlichkeits«-Vorwurf Betroffenen hinaus. Hier sollen offenbar Möglichkeiten angedeutet werden, nach der "fdGO « nunmehr auch das Sozialstaatspostulat als ideologische Staatsschutzwaffe scharf zu machen."

Das von sozialistischen Potenzen gereinigte Sozialstaatsprinzip erscheint denn auch als Inbegriff und verfassungsrechtliche Legitimationsformel einer sich selbst definierenden politischen Mitte: Das sozialstaatliche Prinzip ist, so Klaus Stern, »die weitsichtige Antwort einer Staatsverfassung auf die Probleme einer pluralistischen industriell-technisch-arbeitsteilig gegliederten Massengesellschaft, die die Mitte zwischen dem Prinzip des laissez-faire, laissez-aller und den Konzepten eines nationalistischen oder sozialistischen Totalstaates hält. Das ist kein Sieg einer bourgeoisen Restauration, sondern ein vernünfriger Ausgleich zwischen exzessivem Liberalismus und Kollektivismus sozialistischer oder kommunistischer Provenienz « ${ }^{16}$. Nach einer geläufigen Definition enthält das Sozialstaatsprinzip die Verpflichtung zu sozialer Gerechtigkeit, worunter jenes Verteilungsprinzip verstanden wird, „das jeder Schicht

9 Vgl. nur R. Herzog, a. a. O., Rdn. 60 f.; H. P. Ipsen, Gebundene Sozialstaatlichkeit, in: Festschrift f. K. Zweiger, Tübingen 1981, S. 747 (754 ff.); R. Scholz, Koalitionsfreiheit als Verfassungsproblem, München 1971 , S. 180 ff.; Y. Huh, a. a. O.; H. C. Nipperdey, Soziale Marktwirtschaft und Grundgesetz, Köln 196ı; H. P. Bull, Die Staatsaufgaben nach dem Grundgesetz, Kronberg 1977 S. 179; H. Hablitzel, Wirtschaftsverfassung und Grundgesetz, BayVB! 1981, 69 u. 100 (101); K. Stern, a. a. O., S. 71 1.

10 H. P. Ipsen, Enteignung ..., a. a. O., S. 75 .

11 A. a. O., S. 104.

12 H. P. Ipsen, Gebundene Sozialstaatlichkeit, a. a. O. (Anm. 9), S. 790.

I3 A. a. O., S. 754 ; vgl. zum Positionswandel Ipsens auch J. Gotthold, Wirtschaftliche Entwicklung und Verfassungsrecht, Köln 1975, S. $73 \mathrm{ff}$

is H. P. Ipsen, a. a. O., S. 759 ; wie sich aus dem Zusammenhang (^verfassungsrechtliche Zulässigkeit $*$ ) ergibt, verwechselt Ipsen hier offenbar die Begriffe "legitim* und "legal*.

Is Hierzu im einzelnen M. Kutscha, Verfassung und sstreitbare Demokratie*, Köln 1979, S. 69 ff.

16 K. Stern, a. a. O., S. 692 . 
oder Gruppe der Bevölkerung die ihr zukommenden Rechte einräumt, insbesondere

die wirtschaftliche und kulturelle Lebensfähigkeit auf einem angemessenen Niveau (Standard) « ${ }^{17}$. Trotz ihrer Plattheit und beliebigen Füllbarkeit entspricht diese Definition wegen ihrer status-quo-bewahrenden und "Gruppenansprüche zuerkennenden Inhalte nicht mehr den aktuellen Anforderungen. Die Zunahme staatlicher Lenkungs- und Verteilungsaktivitäten und die Umsetzung von Krisenprogrammen in der Bundesrepublik findet auch in der Fortentwicklung des Verfassungs- und Sozialstaatsverständnisses seine (bewußte) Reflexion und seine (unbewußte) Widerspiegelung ${ }^{18}$. Zwar wäre der Anspruch verfehlt, den Wandel von Verfassungsrecht und -ideologie linear aus den ökonomischen Entwicklungen ableiten zu wollen's. Dennoch eignet sich gerade das Sozialstaatsprinzip als Exempel zur Verdeutlichung des Zusammenhangs zwischen der Geschichte der Bundesrepublik und dem Wandel des Verfassungsdenkens. Wegen seiner begrifflichen und verfassungssystematischen Vagheit, insbesondere aber wegen seiner potentiellen Brisanz für die (verfassungs-) politische Auseinandersetzung ist das Sozialstaatsprinzip stets ein privilegiertes Objekt interpretatorischer Kleinarbeitung gewesen. Da seine verspätete Anpassung an die längst vollzogene Restauration der gesellschaftlichen Herrschaftsverhältnisse in der BRD bereits mehrfach dargestellt und analysiert wurde ${ }^{20}$, können sich die folgenden knappen (und ausführliche Untersuchungen des Themas vielleicht provozierenden) Bemerkungen auf die Phasen der »Planungseuphorie « und der Bewußtwerdung der Permanenz einer tiefgreifenden ökonomischen Krise beschränken. Abschließend soll der Frage der "Nutzbarkeit « dieser Verfassungsformel für die (juristische bzw. rechtspolitische) Argumentation der Linken nachgegangen werden.

\section{Sozialstaatliche Planungseuphorie und ibre Grenzen}

Die bundesdeutsche Staatsrechtslehre tat sich bekanntlich schwer, die staatliche Wirtschaftsplanungs- und -lenkungstätigkeit dogmatisch zu erfassen und auf den (Rechts-)Begriff zu bringen ${ }^{25}$. Nachdem sie jahrzehntelang kaum zur Kenntnis genommen wurde, setzte in den Jahren nach der Krise von 1966/1967, der Novellierung des Art. I09 GG und der Verabschiedung des Stabilitätsgesetzes geradezu ein Boom in der Beschäftigung der Staatsrechtler mit der Planung ein. Dem Sozialstaatsprinzip wurde im Zusammenhang mit der Verpflichtung, den »Erfordernissen des gesamtwirtschaftlichen Gleichgewichts Rechnung zu tragen« (Art. 109 Abs. 2 GG),

I7 Th. Maunz, Deutsches Staatsrecht, 17. Aufl, München 1969, \$ I V.

I 8 Zur Widerspiegelungsproblematik näher $\mathrm{H}$. Wagner, Recht als Widerspiegelung und Handlungsinstrument, Köln 1976.

$19 \mathrm{Vgl}$. hierzu im einzeinen C. U. Schminck-Gustavus, Zur Problematik einer materialistischen Rechtsgeschichte, in: Rechtsgeschichte - Fach ohne Alternative? Bremen 1976, S. I (7 ff.); H. Wagner, Uber einige Schwierigkeiten einer marxistischen Staats- und Rechtstheorie in der Bundesrepublik Deutschland, in: W. Abendroth u. a., Ordnungsmacht? Frankfurt 1981, S. 355 (360 f.).

20 Vgl. Literatur, a. a. O. (Anm. 1).

21 Vgl. aus der Fülle der Literatur nur die von J. H. Kaiser herausgegebene Reihe »Planung«, Baden-Baden I 966 ff.; ferner Gutachten, Referate und Diskussionen auf dem 50. Deutschen Juristentag 1974 zum Thema Welche normativen Anforderungen stellt der Verfassungsgrundsatz des demokratischen Rechtsstaares an die planende staatliche Tätigkeit, dargesteilt am Beispiel der Entwicklungsplanung? \& München 1974; zu den Problemen der Staatsrechtslehre bei der „Bewältigung « der staatlichen Wirtschaftsplanung vgl. im einzelnen: H. D. Fangmann, Staatliche Wirtschaftsplanung und Staatsrechtsideologie, KJ 1972, 1; J. Gotthold, a. a. O (Anm. 13), S. ss ff., 76 ff.; G. Stubv, Diskussionsbeitrag, in: Sitzungsbericht I zum so. DJT, a. a. O., S. 8 s ff.; P. Nahamowitz, Wirtschaftsrecht im nOrganisierten Kapitalismus $4, \mathrm{KJ}$ 1981, $34(46 \mathrm{ff}$ ) 
nunmehr ein Verfassungsauftrag zur staatlichen Globalsteuerung entnommen ${ }^{22}$. Damit stand man allerdings sogleich vor dem Dilemma, eine deutliche Abgrenzung gegenüber der als verfassungswidrig angesehenen Planwirtschaft entwickeln zu müssen. Die überkommene neoliberale Entgegensetzung von Marktwirtschaft und "Zentralverwaltungswirtschaft" reichte hierfür nicht hin. "Es mag zutreffen, daß sich die sozialistischen Staaten des Ostens und die demokratischen Staaten des Westens heute nicht mehr durch Existenz bzw. Nichtexistenz von Planung unterscheiden, weil beide unausweichlich auf Planung angewiesen sind. Trotzdem wird es, wenn die westlichen Demokratien das Gesetz, nach dem sie angetreten sind, überhaupt noch begreifen, entscheidende Unterschiede im , Charakter der Planung geben. Schlagwortartig könnte man sagen, daß Planung im freiheitlichen Sozialstaat eine, Planung zur Freibeit sein muß, oder (...) daß sie zu einem Mehr an Freibeit führen muß «23.

Schon etwas konkreter im Hinblick auf den hier zugrundegelegten Freiheirsbegriff nimmt sich die Definition der Wirtschaftsplanung durch Ipsen aus: Er bestimmt sie als Gesamtheit staatlicher Handlungen "in der Absicht, wirtschaftspolitische Ziele öffentlichen Interesses dadurch zu verwirklichen, daß das Verhalten der privaten Wirtschaft oder die Bedingungen ihrer Marktteilnahme für den Planungszeitraum unter Respektierung, d. h. möglichst geringer Beeinträchtigung ihrer grundrechtlichen Wirtschaftsfreiheit im Zielinteresse beeinflußt werden « ${ }^{24}$. Die Gewährleistung der "Wirtschaftsfreiheit" der Produktionsmitteleigentümer stellt danach also das zentrale Kriterium für die Abgrenzung der Wirtschaftsplanung dar. Ein solches Verständnis der Planung als »Kooperation von Staat und Privateigentum «"s die sich statt umverteilender Eingriffe und Interventionen einer Politik der sindirekten Lenkung" durch Schaffung von (Investitions-)Anreizen etc. bedient, unterscheidet sich nur graduell von den gegenwärtig propagierten Austeritätskonzepten. Letztlich bestimmende Zielgrößen sind in beiden Fällen die Verbesserung der Gewinnaussichten und die Erweiterung der Freiheit für Kapitalstrategien gegenüber ökonomischen und gesellschaftlichen Widerständen ${ }^{26}$.

In der neueren Diskussion wird dabei der Schwerpunkt zum Teil weniger auf den Schutz der subjektiven Rechtsposition des einzelnen Eigentümers als auf den Einbau "des Eigentümers « und »des Unternehmens « in die Verfassungsordnung gelegt ${ }^{27}$. Niklas Luhmann betont: »Nur in seiner spezifischen Rolle als Teilnehmer an der Geldwirtschaft wird der Eigentümer geschützt, und das nicht um seiner Persönlichkeit willen (...), sondern um der Funktionsfähigkeit des Wirtschaftssystems willen $\aleph^{18}$. Es ist danach nur konsequent, die Freiheit des Eigentümers bzw. Unternehmers stets nur als eingebunden in den Zusammenhang der Funktionsfähigkeit des Gesamtsystems zu begreifen - bestimmende Zielgröße bleibt dabei stets die Verbesserung der Chancen für »die Wirtschaft «.

Im Rahmen seiner Kommentierung der "Verfassungsentscheidung für die Sozial-

22 Vgl. K. Stern, Grundfragen der globalen Wirtschaftssteuerung, Berlin 1969 S. 7; ders., Staatsrecht Bd. I S. 703,706 ff.; R. Herzog, a. a. O., Rdn. I6 f.; F. Ossenbühl, Gutachten B zum 5o. DJT, a. a. O., B 149 f.; H. P. Bull, a. a. O., S. 256 f., 426 ff.

23 R. Herzog in: Maunz-Dürig, a. a. O., Rdn. 62; Hervorh. i. Orig.

24 H. P. Ipsen, Rechtsfragen der Wirtschaftsplanung, in: J. H. Kaiser, Planung II, Baden-Baden I966, S. 63 (89); vgl. auch P. Badura, Wirtschaftsverfassung und Wirtschaftsverwaltung, Frankfurt 1971 , S. 117.

25 So W. Leisner, Der Eigentümer als Organ der Wirtschaftsverfassung, DÖV 1975,73 (79).

26 Vgl. R. Hickel, *Haushaltsoperation '82*. Politisch-ökonomische Hintergründe, Bl. f. dt. u. intern. Politik $198 \mathrm{I}, 1062$ (1066 ff.).

27 Vgl. z. B. Referate und Diskussionen zum Thema "Unternehmen und Unternehmer in der verfassungsrechtlichen Ordnung der Wirtschaft* auf der Staatsrechtslehrertagung 1976, VVDStRL 35.

28 N. Luhmann, Grundrechte als Institution, 2. Aufl. Berlin 1974, S. 1. 
staatlichkeit " spricht Herzog daher auch von der verfassungsmäßigen Aufgabe des Staates, "die Wirtschaft aktiv fortzuentwickeln und ihre Existenzchancen zu verbessern "29. Daß dies in einer sich verschärfenden "Wachstumskrise der Industrienationen " auf besondere Probleme stößt, ist in diesem Ansatz bereits mitreflektiert: „Die verfassungsrechrliche Folgerung daraus müßte sein, daß auch ihre Bewältigung Bestandteil einer sozialstaatlichen Politik wäre und ihre verfassungsrechrliche sedes materiae im Sozialstaatsprinzip des Art. 20 I hätte. Wie zahlreiche andere Verfassungsbegriffe ist auch der des Sozialstaates nach Inhalt und Auslegung nicht an jene Situationen gebunden, die beim Inkraftreten des GG vorhanden oder aus geschichtlichen Gründen zumindest bekannt waren. Vielmehr gilt es, den Verfassungsinhalt für grundlegende Situationsveränderungen offenzuhalten « $3^{\circ}$. Damit ist explizit der Anspruch aufgegeben worden, die Verfassung aus dem (festgestellten oder rekonstruierten) Willen des Verfassungsgebers auszulegen; das Sozialstaatsprinzip wird auf die Erfordernisse der Krise hin dynamisiert.

\section{Krisen-Interpretationen und -Ideologien}

Herzog deutet selbst die Richtung an, in der sich eine krisenadäquate Sozialstaatsinterpretation zu entfalten hätte: Wenn die staatliche Sozialpolitik »viel weniger mit der Verteilung von Zuwachsraten als vielmehr mit der Umverteilung des Vorbandenen arbeiten muß, so wird daraus zugleich deutlich, daß das Sozialstaatsprinzip des Art. 20 I nicht einmal die verfassungsrechtliche Garantie des jeweiligen Status quo für alle sozialen Gruppen bewirkt. « $^{31}$ Diese abstrakt formulierte Aussage zielt konkret auf die Legitimation des Abbaus »sozialer Besitzstände « in Gestalt der vielfältigsten sozialversicherungsrechtlichen u. a. Ansprüche. Insoweit wohl am deutlichsten (und ohne die sonst für Staatsrechtler so typische Aversion gegen eindeutige politische Parteinahme) argumentiert Christian Starck in einer neueren Veröffentlichung. Starck beklagt den "Mißbrauch» sozialrechtlicher Ansprüche wie etwa das vorzeitige In-Rente-gehen »unter geschickter Ausnutzung gesundheitlicher Defekte", das »leichtfertige Krankschreiben " durch Ärzte, »gesundheitsschädliche Lebensweise " u. a..$^{32}$. Aus dieser Kritik heraus formuliert Starck den Kantischen kategorischen Imperativ heuristisch auf die gegenwärtige Situation der Bundesrepublik bezogen um: "Kann ich wollen, daß meine Inanspruchnahme sozialer Leistungen (im weitesten Sinne) ein allgemeines Gesetz werde? Und: kann ich wollen, daß mein Verhalten, das zur Inanspruchnahme sozialer Leistungen führt, allgemein gültiges Verhalten ist? Die Fragen sind in einem Sozialstaat deshalb höchst bedeutsam, weil es die Menschen in weitem Maße in der Hand haben, ob sie die Voraussetzungen der gesetzlich normierten Ansprüche erfüllen, z. B. durch vermeidbare gesundheitsschädliche Lebensweise, durch übermäßige Inanspruchnahme des Gesundheitswesens, durch Inaktivität bei der Beschaffung eines Arbeitsplatzes oder durch viele andere Lebensformen bis hin zur bewußten Schröpfung des Staates und der Solidargemeinschaften«. Starck beläßt es nicht bei diesem Entwurf der Elemente eines neuen Bürgerethos, sondern sucht seinen neukantischen Imperativ auch als Handlungsanleitung für den Staat fruchtbar zu machen: "Wie muß der Staat seine soziale

29 R. Herzog, a. 2. O., Rdn. 44

30 A. a. O., Rdn. 15 .

31 R. Herzog, a. a. O., Rdn. 28 - Hervorh. i. Orig.

32 C. Starck, Ist der kategorische Imperativ ein Prinzip des Sozialstaats? ZRP 198i, 97 (99). 
Funktion wahrnehmen, wie muß er insbesondere die Gesetze gestalten, damit die Bürger die oben gestellten Fragen nicht aus dem Sinn verlieren? «33

Mit diesen Gedanken knüpft Starck an ein Sozialverständnis an, dessen wesentliche Züge in den letzten Jahren von Politologen und Verfassungstheoretikern besonders unter dem Stichwort »Krise der Regierbarkeit « ausformuliert wurden. Als eine der zentralen Determinanten für die krisenhafte Gesellschaftsentwicklung erscheint die mangelnde Problemverarbeitungsfähigikeit des Staates angesichts eines stetig wachsenden "Anspruchsdenkens « der Bevölkerung;4. Von den ökonomischen Ursachen wird dabei weitgehend abstrahiert"; ${ }^{35}$ der Schwerpunkt wird teilweise fast ausschließlich auf die subjektive Ebene (und zwar gerade der letztlich am meisten Betroffenen) verlagert. Sinnfällig macht dies etwa der Vorwurf Starcks, der Arbeitslose verschulde seine Lage selbst durch seine geringe Bereitschaft, eine Stelle zu suchen. Die negative Wirkung dieser subjektiven Momente wird sodann aus einem scheinbar objektiven Zusammenhang erklärt: Der Staat könne nur soweit und solange Sozialstaat sein, wie das Bruttosozialprodukt reiche ${ }^{36}$. In der Situation des stagnierenden oder sogar rückläufigen Bruttosozialprodukts räche sich nun, daß der Staat Massenloyalität lange Jahre durch »sozialstaatliche" Geldleistungen erkauft habe: »Alte und starke Impulse der Identifizierung mit dem Gemeinwesen haben ihre Kraft verloren «; wo solche gemeinschaftsorientierten Zustimmungsmotivationen fehlen, muß die $\mathrm{Zu}-$ stimmung »bar bezahlt werden «37.

Diese Deutung enthält ein sehr reales Moment. Unter den Bedingungen der Konjunktur und des Wirtschaftswachstums konnten in der Bundesrepublik während einer langen Periode relativ weitreichende "sozialstaatliche " Leistungen zugestanden bzw. als Instrument zur Systemintegration und zur Verdrängung der - in einer Minderheit nach wie vor virulenten - Gedanken an grundlegende gesellschaftliche Alternativen eingesetzt werden. Ein solches Integrationsmodell läßt sich in Krisensituationen nicht ohne einschneidende Veränderungen weiterführen, bei seiner Umstellung und der Änderung der staatlichen Prioritätensetzung ergeben sich indes erhebliche Legitimitäts- und Loyalitätsprobleme. Diese über den »sozialstaatlichen « Aspekt weit hinausreichenden Problemlagen sind es, die im Zusammenhang des Schlagworts vom "Anspruchsdenken" stets mitbedacht werden. "Was der großen Zahl der Bürger an materieller Verbesserung im Wege der Distribution zuteil wird und dies ist letztlich allein das soziale - das kann ibnen in der demokratischen Staatsform kaum je wieder genommen werden. Wenn ein solcher Versuch unternommen wird, so kann er sich stets nur auf sehr begrenzte Ziele richten, und die Anstrengung, weiche erforderlich ist, um soziale Verteilung wieder rückgängig zu machen, steht in keinem Verhältnis zur Leichtigkeit, mit welcher sozialer ,Fort-

33 Ebenda; Starck unterbreitet selbst entsprechende Vorschläge wie z. B. »Arbeitsverdienst, Leistungen der Arbeitslosenversicherung und Leistungen der Sozialhilfe müssen so deutlich abgestuft werden, daß vor allem regulärer Arbeitsverdienst angestrebt und daß der Sinn der Arbeitslosenversicherung verstanden wird $\propto$ (S. 100).

34 Vgl. z. B. B. Guggenberger, Herrschaftslegitimierung und Staatskrise. Zu einigen Problemen der Regierbarkeit des modernen Staates, in: M. Th. Greven/B. Guggenberger/J. Strasser, Krise des Staates? Darmstadt u. a. 1975, S. 9 (insbes. S. 37); Reiner Schmide, Der geforderte Staat, NJW 1980, 160 ; J. Isensee, Wo etwas fehlt, hilft ein „Grundrecht auf. . $\alpha$, in: FAZ v. 22. 5. 1980 ; sowie die beiden Bände $*$ Regierbarkeit * (Hrsg. W. Hennis/P. G. Kielmansegg/U. Matz), Stuttgart 1977 u. 1979, zur Kritik dieser Theorien vgl. im einzelnen C. Offe, „Unregierbarkeit $\kappa$. Zur Renaissance konservativer Krisentheorien, in: J. Habermas (Hrsg.), Stichworte zur, Geistigen Situation der Zeit،, 1. Bd., Frankfurt 1979, S. 294; E. Lieberam, Krise der Regierbarkeit - ein neues Thema bürgerlicher Staatsideologie, Frankfurt 1977.

35 Vgl, näher C. Offe, a. a. O., S. 308 f.; E, Lieberam, a. a. O., S. $68 \mathrm{ff}$.

36 Vgl. H. P. Ipsen, Gebundene Sozialstaatlichkeit, a. a. O., S. 755 ff.; R. Herzog, a. a. O., Rdn. 23.

37 So P. G. Kielmansegg, Demokratieprinzip und Regierbarkeit, in: Regierbarkeit, Bd. I, a. a. O., S. 125 ; ähnlich C. Starck, a. a. O., S. 101. 
schritt< gelingt « $^{38}$. Die Veränderung der demokratischen Innenausstattung des Staates wird also als Notwendigkeit im Rahmen der Rücknahme sozialstaatlicher Gewährleistungen bereits reflekriert.

Bislang allerdings brauchte die Einsicht, daß der Basiskonsens in der Krise "zunehmend repressiv gewährleistet werden " muß3", in Gestalt manifester Staats-Gewalt nur im Hinblick auf "Randgruppen ${ }^{{ }^{\circ}}$ in die gesellschaftliche Realität umgesetzt zu werden; in Anbetrache möglicher Entwicklungen wird der Ausbau der entsprechenden "Präventiv«-Institutionen und Gewaltapparate jedoch zielstrebig vorangetrieben ${ }^{+t}$. Wenn der Kommentar der FAZ bereits 1980 einen "Abbau des Libertinismus" forderte und verlangte, »die Grenzen der Freiheit . . neu in den Blick zu nehmen « ${ }^{+2}$, so reicht das hier avisierte Spektrum inzwischen von den Nürnberger Massenverhaftungen bis hin zur Entlassung eines "verfassungsfeindlichen " Postbeamten nach drei Jahrzehnten untadeligen Dienstes ${ }^{43}$. Solche und andere Vorfälle belegen anschaulich, daß der Prozeß der "Anpassung " des Sozialstaats an die Bedingungen der Krise seine Parallele in der Verkürzung politischer Freiheitsräume der Staatsbürger findet. Durch unberechenbare staatliche Sanktionen sollen Widerstandsaktionen der Betroffenen gegen die Umsetzung der "Sparpolitik « zum Wagnis werden. Zugleich werden die Gewerkschaften mit Nachdruck auf die Funktion als Ordnungsmacht festgelegt. Ihr Anspruch, den sozialen Besitzstand der abhängig Beschäftigten zu sichern, wird zum "tarifpolitischen Irrweg « gestempelt und ihnen "Verzicht als Konsequenz fehlenden Wachstums « oder die Vereinbarung von »investiven Lohnerhöhungen « anempfohlen ${ }^{+4}$.

Eine zentrale Rolle als präventives Mittel zur Verhinderung der Einsicht in die soziostrukturellen Zusammenhänge und die eigenen Verteidigungsmöglichkeiten spielt freilich eine den Bedingungen der Krise angepaßte Ideologie. Die »Unregierbarkeits «-Theoretiker leiten aus ihren Betrachtungen denn auch häufig die Forderung nach Schaffung eines neuen "geistigen Bewußtseins" in der Bevölkerung ab"s. Nicht die Legitimität des Krisenstaates erscheint als Problem, sondern dessen Legitimation gegenüber der Bevölkerung. Das über viele Jahre hin wirksame Legitimationsmodell "Sozialstaat « für die Bundesrepublik wird verdrängt durch ein anderes: "Die Bundesrepublik war nicht gegründet worden zu dem Zwecke, ihren Bürgern Wohlstand und bequemes Leben zu verschaffen. Sie war gedacht als Schutzbau für einen Teil der Nation, dem es erlaubt war, sich diesen Schutz zu beschaffen « ${ }^{46}$

38 W. Leisner, Demokratie. Selbstzerstörung einer Staatsform? Berlin 1979 S. I $\$ 4-$ Hervorh. von M. K.

39 M. T. Greven, Soziale Probleme und politische Antworten - Sozialpolitische Konzeptionen und Konflikte der siebziger Jahre, in: M. T. Greven/R. Prätorius/T. Schiller, Sozialstaat und Sozialpolitik, Neuwied u. a. 1980, S. 91 ( 118 ).

40 In Wahrheit handelt es sich bei der Zerstörung der natürlichen Umweit, der Bedrohung des Lebens vieler Bundesbürger durch den Betrieb von Kernkraftwerken oder der katastrophalen Lage auf dem Wohnungssektor freilich um alles andere als gesellschaftliche $»$ Randprobleme $\alpha$.

4 I Vgl. im einzelnen J. Seiferr, mInnere Sicherheit *: Risiko für die Demokratie, in: A. Klönne u. a., Lebendige Verfassung - Das Grundgesetz in Perspektive, Neuwied 1981, S. 145; R. Kreissl, Die präventive Polizei, KJ 1981, 128; M. Kutscha/N. Paech (Hrsg.), Im Staat der »Inneren Sicherheit火, Frankfurt $198 \mathrm{I}$.

42 Reißmüller FAZ v. 1. 3. 1980.

43 Vgl. hierzu T. Blanke, Die Radikalisierung der Radikalenverfolgung. Io Jahre Berufsverbotspraxis, KJ 1982, 95 ( $10 \mathrm{r}$ f.); M. Kutscha, Von der Beamten Pflicht zur Verherrlichung des Staates, in: E. Siemantei/H.-D. Wohlfarth (Hrsg.), Der Fall Hans Peter - Entlassung eines $*$ Verfassungsfeindes $\star$, Köln 1982, S. 167.

44 So Rupert Scholz, Sozialstaat. .., a. a. O., (Anm. 8) S. 15.

45 Vgl. Reiner Schmidt, a. a. O. (Anm. 34), S. 163 ; U. Scheuner, a. O., S. 133 . Wesentlich konkreter sind die Thesen des Arbeitskreises Juristen der CSU vom 25. 4. 1982, in denen gefordert wird, der Staat müsse *vor allem mit den Mitteln der Öffentlichkeitsarbeit offensiv werden und bezüglich der Großvorhaben (gemeint ist die Errichtung von Kernkraftwerken etc., M. K.) das geistige Terrain besetzen, bevor es von zerstörenden Kräften beherrscht wird * (Nach *Rechtspolitischer Dienst*, 57. Lfg. v. 30. 4. 1982).

46 G. Gillessen, FAZ v. 31. 5. 1980. 
Die Entsprechung zu den letztlich nur auf den anti-kommunistischen Gegensatz gegründeten Staatslegitimationen wie "Freiheitlichkeit " und "Streitbare Demokratie « bilden jene an den Bürger adressierten Wertpostulate wie "Opferbereitschaft«, "Gemeinsinn «, "Leistungswillen“ etc. Die ideologische Wirksamkeit ${ }^{47}$ dieser dem "Anspruchsdenken « entgegengesetzten Werteformeln beruht auf einer spezifischen Abstraktionsleistung: Das Phänomen der mangelnden Sozialität vieler Bundesbürger wird nicht auf seine sozialen Ursachen zurückgeführt, sondern erscheint als Schuld oder Charaktermangel der Individuen selbst oder als Produkt fehlender Erziehung. Die auf allgemeine Akzeptanz stoßenden abstrakten Forderungen nach einem höheren Maß an mitmenschlicher Solidarität einerseits, Verzichtbereitschaft zugunsten des "Gemeinwesens" andererseits zielen konkret gegen Ansprüche der "sozial Schwächeren", der lohnabhängig Beschäftigten im Zusammenhang mit der Reproduktion der Ware Arbeitskraft. Unter anderem die von der interessierten Presse breit herausgestellten Fälle des "Mißbrauchs des sozialen Netzes dienen zur Legitimation der Verminderung "sozialstaaticher « Leistungen, deren materielle Voraussetzungen die Betroffenen in Zeiten der Konjunktur und Vollbeschäftigung selbst geschaffen haben. Die konkreten haushaltspolitischen Zielprioritäten, zu deren Gunsten die Verzichtsleistungen erbracht werden sollen, bleiben durchweg ausgeblendet.

Nach dem Verständnis der Konservativen soll der Weg zu einem Mehr an »Selbstverantwortlichkeit " und "Eigeninitiative" des Bürgers geebnet werden, zugleich erkennt man jedoch auch in der "Gemeinschaftsgebundenheit « einen Wert von zentraler Bedeutung. Die Werte der "Gemeinschaft" stehen freilich immer dort im Mittelpunkt, wo es um die Legitimation von "Verzicht« und sozialen Opfern der Staatsbürger geht. Der FAZ-Autor Fromme meint in diesem Zusammenhang sogar, das »Volksgemeinschafts «-Denken rechtsextremistischer Kreise gegen den Vorwurf der Verfassungsfeindschaft in Schutz nehmen zu müssen. Schließlich würden nach heutigem Verständnis die Grundrechte »eine Wertordnung errichten, an der sich das Ganze - 'Gemeinschaft ist ein das gleiche meinendes, freilich heute als belastet geltendes Wort - als die Addition der Vielfalt der einzelnen zu orientieren habe. Und gerade der heutige Sozialstaat fordert in vielfacher Weise das Zurückstellen von Individualinteressen hinter dem wie immer von Fall zu Fall definierten Gemeinwohl 4 $^{8}$.

Den abstrakten Forderungen nach "Gemeinsinn ", "Opferbereitschaft " etc. liegt die Fiktion gleichgerichteter Interessen im gesamtgesellschaftlichen Maßstab zugrunde. $\mathrm{Zu}$ "Grundwerten " erhoben und auf Verfassungsebene transformiert, lassen sich derlei Postulate unschwer gegen die grundrechtlichen Gewährleistungen des freien politischen Prozesses ausspielen ${ }^{49}$. Vermittels der Differenzierung zwischen wertverwirklichendem und wertgefährdendem Freiheitsgebrauch kann nunmehr unter Berufung auf die Verfassung der Freiheitsraum für politisch mißliebige Kräfte je nach Opportunität eingeschränkt werden. Wer sich bei seiner Grundrechtswahrneh-

47 Wie weit die Wirksamkeit solcher Formeln reicht, belegt z. B. die Argumentation des sicher nicht als konservativer Theoretiker zu verdächtigenden Fritz Vilmar, der, adressiert an die Bevölkerung, die Aneignung einet »solidarischen Ethik durch die Wiedergewinnung von Gemeinsinn* fordert (In $\mathrm{Zu}-$ kunft: Wachsendes Chaos oder Revolution des Gemeinsinns, in: FR v. 14. 1. 1982).

$4^{8}$ F. K. Fromme, Gewalträtig, ohne Ideologie, knapp bei Kasse, FAZ v. 7. I1. 1980; auch in: Bundesministerium des Innern (Hrsg.), Gewalt von rechts, Bonn 1982, S. 29 (37).

49 Vgl. hierzu im einzeinen: G. Frankenberg, Angst im Rechtsstaat, KJ 1977, 353 (367 f.); E. Denninger, Freiheitsordnung - Wertordnung - Pflichtordnung, JZ 1975, 545; P. Römer, Kritik bürgerlicher Wertauffassungen des Rechts, in: K. Bayertz/H. H. Holz (Hrsg.), Grundwerte. Der Streit um die geistigen Grundlagen der Demokratie, Köln 1978 S. 160; H. H. Holz, werte* contra Demokratie? a. a. O., S. 56; M. Kutscha, Verfassung ..., a. a. O., S. 82 ff. 
mung nicht im Rahmen des konsentierten und damit legitimen Positionsspektrums bewegt, "mißbraucht" seine Grundrechte ${ }^{50}$. Wenn in diesem Sinne etwa ein Grundreche wie die Versammlungsfreiheit "von den Falschen genutzt" wird", bleibt nur noch die Konsequenz, diesen "Falschen", d. h. "Extremisten «, "Verfassungsfeinden « usw. die Berufung auf das Grundrecht zu versagen. Es kann eben, so Ulrich Matz, keine Grundrechte geben, »die dieses grundrechtsgarantierende, ja auf Grundrechten fundierte politische System in Frage stellen « ${ }^{52}$.

An dieser Stelle näher auf die Ansärze zur Verkürzung des verfassungsmäßig gewährleisteten Freiheitsraums einzugehen, erübrigt sich, da diese Thematik bereits häufig Gegenstand kritischer rechtswissenschaftlicher Analyse war. Im folgenden soll die Frage nach den Möglichkeiten gestellt werden, die das Sozialstaatsprinzip im Kontext einer verfassungsrechtlichen Argumentation der "Linken" bieten könnte.

\section{Möglichkeiten und Grenzen einer *alternativen "Sozialstaatsinterpretation}

Angesichts der gegenwärtig betriebenen Haushaltspolitik mit den bekannten Sparmaßnahmen im "sozialen Bereich « liegt die Frage auf der Hand, ob nicht das Sozialstaatsprinzip des Grundgesetzes eine Gewährleistung des erreichten sozialstaatlichen Status quo oder ein Verbor des sozialen »Rückschritts enthalte. In der Tat wird das Sozialstaatspostulat von einigen Autoren in diesem Sinne verstanden ${ }^{33}$. In seiner Essenz wesentlich weiter geht der Ansatz von Helmut Ridder, der der Sozialstaatsklausel ein verfassungsrechtliches Gebot entnimmt, das demokratische Prinzip gleichsam in die "Gesellschaft« hinein zu verlängern: "Ein ssozialer demokratischer Staat ist ein Gemeinwesen, dessen Demokratie nicht auf einen staatlichen< Aufsatz über der Gesellschaft beschränkt ist «. ${ }^{54}$ Wolfgang Abendroth indes erblickt im Sozialstaatsgebot in Verbindung mit Art. Is GG die verfassungsrechtliche Verankerung (nur) der Möglichkeit, die Wirtschafts- und Gesellschaftsordnung in Richtung auf eine inhaltliche Verwirklichung des Gleichheitsgedankens umzugestalten". Dagegen versteht weder Abendroth noch Ridder das Sozialstaatsprinzip im Sinne einer Verfassungsgarantie der erreichten sozialen Besitzstände ${ }^{56}$.

Wie diese kontroversen Positionen zeigen, kann man sich letztlich auch bei der Auslegung des Sozialstaatsbegriffs dem Problem nicht entziehen, inwieweit eine über den Wortlaut der Norm hinausgreifende ("topische«, "rechtsfortbildende" etc.) Interpretation aus der Sicht eines demokratischen Rechtsverständnisses legitim und rechtspolitisch sinnvoll ist. Diese komplexe Problematik ist in den letzten Jahren

50 "Pluralismus entartet zur Anarchie, wenn nicht ein Mindeststandard an ethischer Homogenität vorhanden ist. Zur freiheitlichen Ordnung gehört also ein Bereich des notwendigen Konsenses ebenso wie ein Bereich des legitimen Dissenses* (J. Isensee, Verfassungsgarantie ethischer Grundwerte und gesellschaftlicher Konsens, NJW 1977, 545). *Der Grundkonsens über die Beurteilung der politischen Ordnung, in der wir leben, zerbricht dort, wo Sozialismus und Demokratie gleichgesetze werden « (P. G. Kielmansegg, Ist streitbare Demokratie möglich? in: FAZ v. 2. 6. 1979). „Immer weitere Kreise benutzen die Grundrechte dazu, den gesellschaftlichen Konsens zu zerstören* (C. Starck, Wie Grundwerte zu Unwerten werden können, in: FAZ v. I I. 3. 1978).

¡1 So M. Schreiber (Münchner Polizeipräsident), Duldung von Rechtsbrüchen, in: Die Polizei, Beilage zu Heft $1 / 81$, S. II.

52 U. Matz, Extremisten im öffentlichen Dienst, DÖV I $978,464(467)$.

53 Vgl. z. B. D. Suhr, a. a. O. (Anm. 1), S. 177; H. P. Bull, a. a. O. (Anm. 9), S. 165 f

$\$ 4$ H. Ridder, Nachwort, in: O. E. Kempen, a. a. O. (Anm. 1), S. 235 (247); vgl. auch H. Ridder, Zur verfassungsrechtlichen Stellung der Gewerkschaften im Sozialstaat nach dem Grundgesetz für die Bundesrepublik Deutschland, Stuttgart 1960 sowie in: O. E. Kempen, a. a. O., S. 97

ss W. Abendroth, Zum Begriff ..., a. a. O. (Anm. 2), S. 137

s6 W. A bendroth, a. a. O.; H. Ridder, Nachwort, a. a. O., S. 242. 
zumeist unter den Stichworten "alternative Rechtsauslegung und "Positivismus" diskutiert worden. Namentlich Wolfgang Däubler"7 geht bei seiner Präsentation einer "alternativen « Rechtsinterpretation davon aus, "daß die in der aktuellen juristischen Diskussion zugelassenen Topoi sehr fungibel sind, platter ausgedrückt, daß man lege artis außerordentlich vielfältige Ergebnisse begründen kann ${ }^{{ }^{8}}$. Dementsprechend wird die vorherrschende Interpretationsmethodik gleichsam mit umgekehrter Stoßrichtung zum Einsatz gebracht: Der "alternativen Dogmatik « geht es, so Däubler, darum, »daß die wünschbaren, den Interessen der Lohnabhängigen entsprechenden Rechtsinhalte im Wege gängiger dogmatischer Argumentation begründet werden, in einer Form also, die wenigstens eine Kommunikation mit den Entscheidungsträgern ermöglicht « '. In Anwendung seiner Methode entnimmt Däubler etwa dem im Grundgesetz verankerten Prinzip der Menschenwürde ein umfassendes Recht auf Mitbestimmung der lohnabhängig Beschäftigten im Betrieb ${ }^{60}$.

Insoweit ein Ansatz zuvörderst auf Teilnahme am Diskussionsprozeß innerhalb des »anerkannten « Meinungs- und Autorenspektrums und auf Herstellung eines Konsenses auf der Basis gemeinsamer Topoi und Wertprämissen aller Beteiligten abzielt, birgt er bestimmte Risiken: Das Problem der Durchsetzung von Rechtspositionen in der sozialen Realität kann dann letztlich allein als eine Frage des rational-fachwissenschaftlichen Diskurses, der argumentativen Überzeugung der wherrschenden Meinung und der Gerichte erscheinen. Die juristische Argumentation tritt damit nicht mehr neben, sondern an die Stelle der gesellschaftlichen Praxis. Wenn die Illusion gemeinsamer Wertprämissen der an der Auseinandersetzung Beteiligten gezüchtet und der Zusammenhang zwischen Rechtsentwicklung und sozialer Machtkonstellation ausgeblendet wird, kann eine "alternative“ Rechtsauslegung zur Entpolitisierung derer beitragen, deren Interessen zu vertreten ihre erklärte Absicht ist.

Die Kritik an der »alternativen Rechtsauslegung " richtet sich auch dagegen, daß diese die Usurpation gesetzlich nicht explizit eingeräumter "Auslegungs«-Spielräume durch »h. M.« und "Richterrecht« nicht grundsätzlich ablehnt ${ }^{61}$. Verwiesen wird auf die vielfältigen negativen Erfahrungen mit der Interpretation praeter und contra legem, aktuell etwa der Überlagerung der verfassungsmäßigen Koalitionsfreiheitsgarantie durch eine »Arbeitskampfordnung oder der Relativierung der grundrechtlichen Freiheiten durch ein allgemeines Verfassungsprinzip der "streitbaren Demokratie«. - Als adäquate Antwort hierauf postulieren namentlich Helmut Rid$\operatorname{der}^{6_{2}}$ und Peter Römer ${ }^{\sigma_{3}}$ einen "Positivismus«, der auf der strikten Verteidigung der

\$7 W. Däubler, Gesellschaftliche Interessen und Arbeitsrecht, Köln 1974, S. 43 ff,; ders., Einige Überlegungen zur alternativen Rechtsinterpretation, in: P. Römer (Hrsg.), Der Kampf um das Grundgesetz, Frankfurt 1977, S. 33; zur Kontroverse in Verfassungsrecht vgl. die Diskussion in: P. Römer, a. a. O., S. $171 \mathrm{ff}$.

s8 W. Däubler, Gesellschaftliche Interessen, a. a. O., S. 52.

59 A. a. O., S. 51/52.

60 W. Däubler, Das Grundrecht auf Mitbestimmung und seine Realisierung durch tarifvertragliche Begründung von Beteiligungsrechten, Frankfurt 1973. In einer neueren Arbeit hat Däubler allerdings herausgestellt, daß das Grundrecht auf Mitbestimmung nicht einen einkjagbaren Anspruch auf Einführung der Montanmitbestimmung in allen Großbetrieben zum Gegenstand habe, sondern als ^Ermöglichungsrecht* lediglich die verfassungsmäßige Absicherung der Chance zur politischen Durchsetzung dieser Forderung darstelle (Gewerkschaften und Arbeitsrecht, in: R. Hegenbarth/W. Kaupen/U. Reifner (Hrsg.), Der Gebrauch von Recht (Arbeitsmaterialien zum 20. Deutschen Soziologentag), Bochum 1980, S. $30(38))$.

61 Vgl. P. Römer, Diskussionsbeitrag, in: ders., a. a. O. (Anm. 57), S. 211

62 H. Ridder, Negativkonvergenzen? Polemisches zum selektiven Anti-Positivismus, in: Festschrift für E. Rabofsky, Köln 1976, S. 46 . Auf der anderen Seite hat Ridder aber gerade im Hinblick auf das hier in Rede stehende Thema die Feststellung getroffen, daß bestimmte Vorstellungen des Verfassungsgebers in bezug auf das Sozialstaatspostulat snach den allgemein anerkannten juristischen Auslegungsgrundsätzen einer weitergreifenden Exegese nicht im Wege stehen * (Zur verfassungsrechtlichen Stellung ..., a. a. O., S. 112; Hervorh. von mir, M. K.). 
Normativität des positiven Rechts insistiert und sich gegen die Aufweichung der (Verfassungs-)Normen durch "Richterrecht " bzw. eine sich desselben Instrumentariums bedienende "alternative " Interpretation wendet. "Wenn es nicht gelingt ", fragt Römer, »die Interessen der lohnabhängig Arbeitenden im Gesetz selbst festzuschreiben, ist es dann nicht illusionär zu glauben, sie im Wege alternativer Interpretation durchsetzen zu können, obwohl doch der Einfluß der lohnabhängig Arbeitenden auf Justiz und Verwaltung viel geringer ist als auf das Parlament? « ${ }^{64}$ Der Appell an den Gesetzgeber erhält damit im Verständnis der "positivistischen « Vertreter einen zentralen Stellenwert. Die gegenwärtigen politischen Verhältnisse geben für einen "Parlamentsoptimismus “6s allerdings wenig Anlaß; letztendlich unterscheiden sich die in Justiz und Gesetzgebung dominierenden Interessenpositionen kaum.

Auf der anderen Seite bleibt ein weit fortgeschrittener Zersetzungsprozeß der traditionellen Legalitätsordnung und ein Machtzuwachs der Justiz zu konstatieren, der die pessimistische Frage provoziert, ob nicht die von "positivistischer "Seite befürwortete Strategie der "Rekonstruktion der Legalordnung« einen Kampf gegen Windmühlenflügel darstelle ${ }^{66}$.

Eine einseitige Ausrichtung auf die Parlamentsebene würde ignorieren, daß sich die Herausbildung von Positionen in der Rechtsprechung keineswegs abgekoppelt von der gesellschaftlichen Entwicklung vollzieht: Zumeist in den unteren Instanzen der Justiz manifestiert sich mitunter durchaus ein höheres $M a ß$ an Sensibilität gegenüber bestimmten Forderungen und Anliegen der Bevölkerung als in der Gesetzgebung erwähnt sei hier nur die Entscheidungspraxis von Arbeitsgerichten zur Zulässigkeit der Aussperrung ${ }^{67}$, zur Berufsverboteproblematik ${ }^{68}$ oder von Verwaltungsgerichten zur Genehmigung von Atomkraftwerken ${ }^{69}$. Die Gegensteuerungsversuche reichen denn auch von informellen Kontakten zwischen Vertretern der verschiedenen oberinstanzlichen und Bundesgerichte zwecks Herstellung einer einheitlichen Rechtsprechung ${ }^{70}$ bis zur Verkürzung des Rechtsschutzes durch Verfahrensänderungen ${ }^{71}$ oder die Anerkennung eines gerichtlich nicht überprüfbaren "Beurteilungsspielraums * der Einstellungsbehörden ${ }^{72}$.

63 P. Römer, Kleine Bitte um ein wenig Positivismus, in: ders. (Hrsg.) Der Kampf ..., a. a. O. (Anm. 57), S. 87; vgl. ferner H.-E. Böttcher, Zum Selbstverständnis gewerkschaftlich organisierter Richter und Staatsanwälte, KJ 1981, 172 (176 f.); H. Wagner, Über einige Schwierigkeiten..., a. a. O. (Anm. 19), S. 365 ff.; R. Geffken, Seeleutestreik und Hatenarbeiterboykott, Marburg 1979, S. 8 ff.

64 P. Römer, a. a. O., S. 93.

6s Dies hält D. Grimm (Reformalisierung des Rechtsstaats als Demokratiepostulat? JuS 1980, 704 ff., 709) I. Maus entgegen.

66 So H.-E. Böttcher, a. a. O., S. 177.

67 Vgl. z. B. ArbG Frankfurt NJW 1979, 388; ArbG Stuttgart DuR 1979, 86; LAG Frankfurt NJW 1979, 2268 .

68 Vgl. die Nachweise bei G. Frankenberg, Staatstreue. Die aktuelle Spruchpraxis zu den Berufsverboten, KJ 1980, 276; N. Paech/H. Stein, Radikale vor Gericht, DuR 1979, 416; K. Böwer, Die Entwicklung der Berufsverboterechtsprechung der Arbeitsgerichte in NRW - Erfahrungen aus Anwaltssicht, DuR 1981, 276.

69 Hierzu im einzelnen K. Busselmann, Grundrechtsschutz und Reform des atomrechtlichen Genehmigungsverfahrens, KJ i980, 389 .

70 So fand Berichten zufolge im November 1979 zwischen Richtern der mit Berufsverbotsverfahren befaßten Senate des BVerwG und des BAG sowie einigen Präsidenten von Landesarbeitsgerichten und Oberverwaltungsgerichten ein „Gespräch* statt; vgl. R. Wahsner/N. Paech, Berufsverbote und kein Ende! DuR 1980,417 (418).

71 Der von der Bundesregierung eingebrachte Gesetzentwurf für eine Verwaltungsprozeßordnung sieht u. a. die erstinstanzliche Zuständigkeit der Oberverwaltungsgerichte für Großverfahren vor; diese Begrenzung auf eine Tatsacheninstanz wird u. a. mit den bisherigen nolkswirtschafelich teilweise schlechthin unvertretbaren Verzögerungen bei der Errichtung solcher Großanlagen « begründet (nach »Rechtspolitischer Dienst $\propto$, 58. Lfg. v. 7. 5. 1982). Zur Änderung des atomrechtlichen Genehmigungsverfahrens näher K. Bosselmann, a. a. O.

72 Vgl. z. B. BAG NJW 1981, 71; BVerwG NJW 1981, 1386; hierzu im einzelnen M. Kutscha, Die Problematik eines *Beurteilungsspielraums * bei einer Einstellung, BIStSozArbR $1981, t 6 t$. 
Ebenso wie die "alternative " Auslegung geht auch der "positivistische" Ansatz davon aus, daß jede Norm einen Rahmen markiert, der unterschiedliche Konkretisierungsmöglichkeiten zuläßt ${ }^{\text {s }}$ und damit sowohl die Position einer wherrschenden Meinung" als auch eine "alternative" Position abdecken kann. In diesem Zusammenhang stellt sich freilich die Frage, ob es einen objektiven Norminhalt und -rahmen gibr, der unabhängig von der jeweiligen Norminterpretation besteht. Die Antwort kann sich nur aus der Bestimmung der sozialen Spezifik rechrlicher Normierung ergeben: Die am Gesetzgebungsprozeß Beteiligten repräsentieren gesellschaftliche Gruppen und Klassen, die um die Durchsetzung ihrer Interessen miteinander streiten; die Rechtsnorm ist das kompromißhafte Produkt dieser Auseinandersetzung. Der Norminhalt ist damit durch den der Entstehung zugrundeliegenden und sich im Gesetzeswortlaut niederschlagenden Kompromiß festgelegt, dieser bildet den »Fixpunkt " ${ }^{74}$ für die Bestimmung des objektiven Sinns der Norm. Wir können, wie Wolfgang Abendroth bemerkt, "den objektiven Sinn der Normen des jeweiligen Klassenkompromisses durchaus objektiv feststellen und erkennen, wenn wir ihn exakt sozialhistorisch überprüfen und von hier aus seine Wortbedeutung und die Grenzen ihrer Auslegungsfähigkeit bestimmen «"s. Nur durch die Rückbeziehung der Rechtsnorm auf den demokratischen Gesetzgeber läßt sich danach der Spielraum für ihre Interpretation bestimmen.

Es bleibt die Frage, ob das »klassisch" juristisch-dogmatische Instrumentarium zur Erkenntnis des Auslegungsrahmens einer - als Niederschlag eines sozialen Kompromisses verstandenen - Gesetzesnorm hinreicht. Äußerungen von Vertretern des "positivistischen « Ansatzes wie etwa die Forderung Ridders nach "Wiederherstellung einer anständigen Lehre von der Rechtsanwendung in der Juristenausbildung ${ }^{76}$ legen dies als Schlußfolgerung nahe und lassen Vorbehalte gegen eine sozialwissenschaftliche Ausbildung yon Juristen anklingen. Eine Beschränkung auf das herkömmliche Instrumentarium der Rechrsinterpretation erscheint indes kaum als geeignet, die soziale Einbindung juristischer Praxis sichtbar zu machen und einer inhaltlichen Verschiebung der Normaussage nach Maßgabe gesellschaftlich vorherrschender Interessen zu wehren - diese sozialen Interessen bleiben unerkannt. Die Ideologie der gesellschaftlichen Neutralität von Recht und Rechtsanwendung kann sich umso unangefochtener entfalten.

Eine rationale Analyse der Tendenzen und Entwicklungen im Recht kann nur eine Rechtswissenschaft leisten, die sich auf die Erkenntnis sozialer Prozesse gründet, nicht dagegen ein blinder Positivismus. Auf dieser Grundlage kann auch erst die Auflösung des klassisch-bürgerlichen Rechtsbegriffs und die Verdrängung der gesetzgeberischen Entscheidungsinhalte durch richterliche "Rechtsfortbildung" als Widerspiegelung geselischaftlicher Prozesse erkannt und damit rational »begriffen « werden.

Eine sich als alternativ verstehende Rechtswissenschaft kann aus dieser Sicht die soziale Interessenbindung einer sich contra legem bewegenden $\gg$ h. M.» aufdecken und eine sich innerhalb des normativen Rahmens bewegende Position argumentativ verteidigen. Stets bleibr jedoch der Zusammenhang zwischen gesellschaftlicher Praxis und Rechtsentwicklung, die $*$ Dialektik von außerjuristischem und juristischem Kampf «"7 deutlich zu machen. Wenn eine Norm für die jeweils vertretene Interes-

73 So bereits H. Kelsen, Reine Rechtslehre, 2. Aufl. Wien 1960, S. 348; vgl. auch P. Römer, Kleine Bitte ..., a. a. O., S. 94 .

74 P. Römer, Verfassungssubstanz und demokratischer Prozeß, DuR 1976, 346 (348).

75 W. Abendroth, Diskussionsbeitrag, in: P. Römer (Hrsg.), Der Kampf ..., a. a. O., S. $227 / 228$.

76 H. Ridder, Diskussionsbeitrag, in: M. Kittner (Hrsg.), Streik und Aussperrung, Frankfurr 1974, S. 534 ( 536$)$.

77 H. Wagner, Über einige Schwierigkeiten ..., a. a. O. (Anm. 19), S. 366. 
senposition nichts "hergibt", bleibt nur die Forderung nach legislativer Ände-

rung.

Von dieser Position aus ist auch der verfassungsrechtliche Inhalt des Sozialstaatsgebots zu bestimmen. Weder Entstehungsgeschichte noch Wortlaut und Stellung im Gefüge der Verfassung bieten eine Grundlage für Interpretationen, die sich die Erfüllung des Sozialstaatspostulats nicht anders denn durch die Verbesserung der Gewinnchancen der Unternehmen durch entsprechende staatliche Umverteilungsmaßnahmen vorstellen können. Die ständig wiederholte These, daß höhere Gewinne mehr Investitionen und damit mehr Arbeitsplätze bedeuten, wird bereits durch die Zahlenangaben von Unternehmerseite widerlegt: Während die privaten Netrogewinne von 1970 bis 1980 im Jahresdurchschnitt um 7,4\% stiegen, nahmen die Nettoinvestitionen im gleichen Zeitraum nur um durchschnittlich $4,2 \% \mathrm{zu}$. Wurden 1971 noch $64 \%$ der Nettogewinne reinvestiert, waren es 1980 nur noch $52 \%{ }^{78}$. Zugleich wird ein immer größer werdender Anteil der Investitionen zur Rationalisierung und damit zur Einsparung von Arbeitsplätzen verwendet. Während 198 I noch 4 I von 100 befragten Industrieunternehmen die Rationalisierung als Hauptziel ihrer Investitionen bezeichneten, waren es 1982 bereits 53 .

Im Zusammenhang mit der Sozialisierungsermächtigung des Art. Is GG ist das Sozialstaatsgebor Ausdruck der Kompromißentscheidung des Verfassungsgebers hinsichtlich der künftigen Sozial- und Wirtschaftsordnung. Mit Recht hat Wolfgang Abendroth betont, daß der verfassungsmäßig formulierte Sozialstaatsgedanke als Gegengewichtsfaktor verhindern soll, daß der Grundrechtsteil des Grundgesetzes als starre Garantie der bestehenden Gesellschafts- und Wirtschaftsordnung mißverstanden wird ${ }^{79}$. Eine Verfassung, die mit Art. is GG selbst die Transformation in eine Wirtschaftsordnung sozialistischer Prägung gestattet, legalisiert damit zugleich auch weniger gravierende Eingriffe des Staates in den Wirtschaftsprozeß ${ }^{\text {so }}$. Der Sozialstaat ist also keineswegs auf "marktkonforme « Aktivitäten beschränkt, die Verfassung ermächtigt ihn vielmehr zu einer Wirtschafts- bzw. Sozialpolitik mit gänzlich anderen Zielprioritäten.

Damit bleibt freilich die Frage noch unbeantwortet, ob sich unter bestimmten Voraussetzungen aus dem Sozialstaatsgebot eine verfassungsrechtliche Verpflichtung des Staates zu konkreten wirtschaftspolitischen Maßnahmen ergibt. Denkbar wäre die Annahme, daß sich in der Situation einer massiven Gefährdung bzw. eines Abbaus des sozialen Besitzstandes einer großen Anzahl von Bürgern das Sozialstaatspostulat gleichsam zur Pflicht des Staates "verdichtet ", in einer bestimmten Weise zu intervenieren (Beispiel: Verstaatlichung des AEG-Konzerns). Die verfassungsrechtliche Ableitung der konkreten Voraussetzungen für eine solche »Verdichrung « des Sozialstaatspostulats wie auch die Bestimmung der hierdurch gebotenen konkreten staatlichen Reaktionsformen dürften indes erhebliche Schwierigkeiten bereiten ${ }^{81}$ - auch der Entstehungsgeschichte des Grundgesetzes läßt sich insoweit nichts entnehmen.

Aber wäre es nicht auch denkbar, mit der Interpretation des Sozialstaatsbegriffs eingefahrene Wege, hier die Kategorie einklagbarer subjektiv-öffentlicher Rechte zu verlassen und das Sozialstaatsgebor als (nichtjustiziablen) kollektiven Anspruch der

\footnotetext{
78 Angaben nach: Arbeitsgruppe Memorandum, Alternativen der Wirtschaftspolitik, Memorandum '82, in: Blätter f. dt. u. intern. Politik $1982,617(619)$.

79 W. Abendroth, Zum Begriff ..., a. a. O., S. 116.

80 Vgl. im einzelnen H. Ridder, Die soziale Ordnung des Grundgesetzes, Opladen 1975, S. 94 ff.; G. Stuby, Der Eigentumsbegriff des Grundgesetzes und seine normativen Anforderungen für die Gegenwart, DuR 1974, 157 (177 t.); N. Reich, Markt und Recht, Neuwied 1977, S. 92 f.; K.-J. Bieback, Sozialstaatsprinzip und Grundrechte, Ms. Hamburg 1982 (erscheint demnächst).

81 Vgl. hierzu K.-J. Bieback, a. a. O.
} 
Lohnabhängigen zu begreifen, wie es teilweise in der Diskussion um das Recht auf Arbeit geschieht? Hier verschwimmt dann freilich die Grenze zwischen rechrlicher Verbürgung und politischer Forderung. Aber hat die Sozialstaatsformel nicht ohnehin ihre größeren Potenzen im Bereich der politischen Auseinandersetzung? Die politisch-legitimatorische Sprengkraft dieser und anderer Verheißungen (Gebote, Aufträge) wird von konservativer Seite denn auch deutlich gesehen: Herzog warnt davor, daß der "Sozialstaat « zum »archimedischen Punkt einer ideologisch ausgerichteten Umwertung der Verfassungswerte« werden könne ${ }^{\beta_{2}}$, und Isensee konstatiert: »Das soziale Staatsziel ist unersättlich und uneinholbar. . . Die unerfüllte und unerfüllbare Verfassung wird zum Rechtstitel für Revolutionäre«..$_{3}{ }^{8_{3}}$

Nun, momentan freilich geht es um viel weniger: die politische Verteidigung sozialer Errungenschaften gegen deren Abbau.

Martin Kutscha

\title{
Ein Richter als Anwalt der Unterprivilegierten
}

\author{
William Douglas: Gegenbild zur konservativen Justiz
}

Das Bild, das die Vereinigten Staaten von Amerika zur Zeit bieten, scheint sich verändert oder gar verzerrt zu haben. Der Geist der Gründerväter, der großen Aufklärer und Humanisten Jefferson und Madison, der Geist der Unabhängigkeitserklärung, der Virginia Bill of Rights von 1776 , der Bundesverfassung von 1789 und 179I, wonach alle Menschen gleich frei seien, und wo bestimmt ist, daß der Kongreß kein Gesetz machen dürfe, das diese Freiheit einschränke, und wo eine strenge Trennung des Staates von religiösen Dingen verfügt ist - dieser radikale Geist scheint verdunkelt. Solche Verdunkelungen hat es allerdings schon mehrfach im öffentlichen Leben und in der Rechtspflege dieses Gemeinwesens gegeben. Schon in seinem Anfang lag der Schatten der versklavten Neger darauf; heute ist die Regierung der Vereinigten Staaten zum Protektor von grausamen Militärdiktaturen geworden. Diese Schatten treffen uns umso stärker, als der kulturelle Einfluß Amerikas auf vielen Sachgebieten und auf hohen, mittleren und unteren Ebenen seit dem zweiten Weltkrieg bei uns und im ganzen europäischen Westen, ja sogar überall, ungemein stark ist. Man könnte statt von Einfluß geradezu von Überflutung sprechen; und sicherlich ist diese kulturelle Überflutung gerade jener Freiheit, Toleranz und Mannigfaltigkeit zu verdanken, die in der amerikanischen Verfassung vorgebildet sind. Diese Gleichheit in der Freiheit war schon das große Erlebnis des französischen Aristokraten Alexis de Tocqueville bei seiner Amerika-Reise im Jahre I 832 . Und deshalb war dieses Land das Ziel und die Zuflucht von Menschen aus aller Welt vor Not, Armut und Unterdrückung. Das gab es zwar auch in Amerika selber, nicht nur

82 R. Herzog, a. a. O., Rdn. 3. Eine sehr eindeutige (und für die Betroffenen oftmals folgenschwere) Kategorisierung nimmt K. D. Bracher vor, der die „Überstrapazierung der Verfassungsnormen « durch die *von links vorgebrachte Kritik an der angeblichen ,Nichterfüllung، des Grundgesetzes* wie auch die *rigorose Konfrontation von Norm und Wirklichkeit* als *verfassungsfeindlich* bezeichnet, weil sie gegen die Existenz und Funktion des Verfassungssystems selbst wirken könne (Bewährung und Anfechtung: Zum Streit um Demokratie und Verfassung in der Bundesrepublik, in: M. Funke (Hrsg.), Extremismus im demokratischen Rechrsstaat, Bonn 1978, S. 422 (425).

83 J. Isensee, Wo etwas fehlt ..., a. a. O. (Anm. 34); auch W. Leisner klagt in einer Bestandsaufnahme der Verfassungsentwicklung (Flexibilität als Bewährungsprobe? BayVBl 1979, 518 ), daß es nicht gelungen sei, den vom Grundgesetz geschaffenen Rahmen für *Regimeveränderungen* einzuengen. 